\title{
Perceção de homofobia e discriminação da comunidade LGBTQIA+ no contexto da disciplina de Educação Física em Portugal
}

\section{The perception of homophobia and discrimination of LGBTQIA+ community in the context of Physical Education in Portugal}

\author{
Ana Catarina Craveiro (1) \\ José Mendes (2) \\ Fernando Vieira $(1,3,4)$ \\ (1) Instituto Piaget (ISEIT), Almada, Portugal \\ (2) INTELECTO - Psicologia \& Investigação, Ponta Delgada, Ilha de São Miguel, Açores, Portugal \\ (3) Instituto Piaget (ISEIT), Kinesiolab - Laboratory of Human Movement Analysis, Portugal \\ (4) Research in Education and Community Intervention (RECI), Portugal
}

Recebido: 21/10/2021; Revisto: 04/11/2021; Aceite: 09/11/2021.

\begin{abstract}
Resumo
Contexto e Objetivo: O presente estudo procurou explorar a homofobia internalizada e sentimento de discriminação da comunidade LGBTQIA+ (Lésbica, Gay, Bissexual, Transsexual, Queer, Intersexo, Assexual) no contexto da disciplina de educação física. Métodos: Estudo quantitativo, qualitativo e exploratório numa amostra por conveniência, não probabilística, realizado entre maio e julho de 2021. Participaram no preenchimento de um questionário sociodemográfico, questionário de conforto com a identidade sexual (Escala de Homofobia Internalizada), e Escala de Discriminação Quotidiana 180 indivíduos com idades compreendidas entre os 18 e os 60 anos. Resultados: Na avaliação da homofobia internalizada, a perceção de estigma externo apresentou correlações moderadas e altas com as variáveis associadas à discriminação em meio escolar, inclusive na disciplina de educação física. Foi possível verificar diferenças estatisticamente significativas nas modalidades preferidas praticadas na educação física entre os sexos, onde as raparigas preferiram modalidades coletivas e de contacto, enquanto os rapazes optaram por modalidades individuais sem contacto. Através da análise qualitativa, foi possível verificar memórias de discurso homofóbico por parte dos colegas aquando da prática da disciplina de educação física. Conclusões: Revela-se necessário uma maior compreensão sobre as diferenças sexuais e a prática das modalidades da disciplina de educação física. Emergem preocupações associadas ao abandono da prática de exercício físico ou desporto devido à discriminação sentida pelos jovens. Estudos futuros devem analisar a perspetiva dos intervenientes na elaboração do currículo de educação física, tendo em consideração a evolução da sociedade.
\end{abstract}

Palavras-Chave: Discriminação; Educação Física; Homofobia interna; LGBTQIA+; Estudo misto.

\section{DI\&D | ISMT}

rpics@ismt.pt

https://rpics.ismt.pt
Ana Catarina Craveiro 


\begin{abstract}
Background and Aim: The aim of this study was to investigate internalized homophobia and the feeling of everyday discrimination among LGBTQIA+ (Lesbian, Gay, Bisexual, Transsexual, Queer, Intersex, Asexual) community in physical education. Methods: Qualitative, quantitative and exploratory study was used on a convenience sample, nonprobabilistic, carried out from May 2021 to July 2021. One hundred eighty individuals, aged between 18 and 60 , participated in the study by answering a sociodemographic questionnaire, a questionnaire evaluating comfort with sexual identity (the Internalized Homophobia Scale), and the Everyday Discrimination Scale. Results: Regarding the evaluation of internalized homophobia, the perception of external stigma presented moderate to high correlations with the variables associated with discrimination in school settings and physical education classes. It was possible to confirm statistically significant sex differences in favorite sports practiced in physical education classes, where girls preferred group contact sports and boys preferred individual/non-contact sports. Through qualitative analysis was noticeable that most participants recalled homophobic rhetoric from classmates during physical education classes. Conclusions: There is a need for a greater understanding of gender differences and physical education practice. Concerns arise associated with the abandonment of physical exercise or sport due to the discrimination felt by young students. Future studies should analyze the perspective of those involved in the development of the physical education curriculum, taking into account the evolution of society.
\end{abstract}

Keywords: Discrimination; Internal homophobia; LGBTQIA+; Physical education; Mixed study.

\title{
Introdução
}

Homens e mulheres enfrentam muitas vezes um conflito de sentimentos que se relacionam com a sua sexualidade (Pereira \& Leal, 2005), em que a distorção do autoconceito, muitas vezes influenciado pelo contexto social, se associa à diminuição da saúde mental de jovens LGBTQIA+ (Lésbicas, Gays, Bissexuais, Transsexuais, Queers, Intersexo, Assexuais e outros) (Hossain \& Ferreira, 2019). Uma visão histórica sobre o conceito da homossexualidade mostra que a homossexualidade era considerada uma patologia até à terceira edição do Manual Diagnóstico e Estatístico de Perturbações Mentais (Diagnostic and Statistical Manual of Mental Disorders - DSM-III), tendo-se mantido este rótulo por mais de 20 anos (Barounis, 2017; Drescher, 2015). Borrillo (2009) alertou para o facto de a homossexualidade ter passado de pecado a crime e de crime a doença por mais de dois séculos.

A consciencialização ou autoperceção da orientação sexual surge ao longo da vida, conseguindo alguns indivíduos afirmar a sua sexualidade na adolescência, enquanto outros somente o conseguem fazer no período da adultez (Macedo, 2018). Por outro lado, Landi et al. (2020) mencionam a existência de muita sensibilidade sobre a temática LGBTQIA+ e defendem que os jovens muitas vezes não se sentem preparados para se exporem e assumirem a sua orientação sexual perante a sociedade em que se inserem. A American Psychology Association (APA) define a orientação sexual, a atração romântica e sexual entre duas pessoas do mesmo sexo (APA, 2012). Neste sentido, revela-se pertinente uma melhor compreensão sobre a evolução do conceito de género, uma vez que a sua determinação e construção tem-se definido com a evolução da sociedade (Gonçalves \& Gonçalves, 2021). Portanto, considera-se que o género é um constructo social e cultural que se diferencia pelos atributos dos homens e mulheres, meninos e meninas, variando este conceito em diferentes contextos culturais (APA, 2012; UNICEF, 2017). 
Devido a determinados sistemas de opressão e forças de poder, a comunidade LGBTQIA+ pode experienciar no seu quotidiano discriminação e isolamento (Parmenter et al., 2021), em que muitas das dificuldades se relacionam com a dimensão da abertura da homossexualidade perante o outro (Pereira \& Leal, 2005). Na opinião de Berg e Kokkenen (2021) tais obstáculos e discriminação na comunidade LGBTQ+ estão muitas vezes presentes na escola, principalmente na disciplina de educação física.

Apesar do reconhecimento destas dificuldades, Landi et al. (2020) alegam a existência de muitas objeções na abordagem de estudos LBGTQIA+ na comunidade escolar. Essas dificuldades (e.g., discriminação) podem dever-se ao facto de se utilizar terminologia discriminatória (e.g., "não sejas bicha", "isso é tão gay", entre outras) para com a comunidade LGBTQIA+ no contexto escolar (Nadal et al., 2011). Estas expressões, na visão de Woodford et al. (2013), passam uma mensagem negativa e destrutiva para os membros da comunidade LGBTQIA+, porque apesar de ser ou não intencional, estes comentários levam ao sentimento de inferioridade e desprezo.

Na sociedade, a educação sexual e afetiva dos gays e lésbicas era feita, sobretudo, através de recursos muitas vezes ocultos (e.g., recursos literários, cinematográficos, culturais) (Borrillo, 2009). Este autor revela uma realidade encoberta e dissimulada, em que se desenvolviam sentimentos como o da negação em relação à própria orientação sexual ou a falta de conhecimento.

Existem evidências na literatura que apontam para a existência de homofobia nas escolas, muitas vezes reproduzida em materiais didáticos (Santos \& Cerqueira-Santos, 2020). Os autores defendem ainda que estes indivíduos podem criar um sentimento de homofobia internalizada, que consiste na dificuldade de autoaceitação que podem originar preconceitos individuais e intolerância para com a homossexualidade na sociedade.

Perante a lacuna de falta de informação sobre a comunidade LGBTQIA+, a associação de jovens LGBTQI e apoiantes (rede ex aequo), tem desenvolvido sessões de esclarecimento nas escolas no intuito de fazer frente à desinformação e discriminação ainda presentes no ensino português (Rocha et al., 2019). A educação sexual tem sido marcada por avanços e retrocessos nas políticas educacionais (Santos, 2017). Beserra et al. (2019) revelaram que as aulas de educação física são um local onde muitas vezes acontecem episódios de bullying homofóbico. Justificam ainda o facto anterior, à falta de maturidade dos estudantes, à exposição e à interação social características da disciplina de educação física. Na opinião de Devís-Devís et al. (2018), estes episódios de discriminação não acontecem somente em aulas de educação física, mas também nos balneários, locais onde os alunos e alunas queer se sentem muitas vezes intimidados e inferiorizados, tornando-os um alvo fácil. Estes acontecimentos de discriminação, podem levar ao desinteresse ou abandono da prática de atividade física (Symons et al., 2010). Por outro lado, Herrick e Duncan (2020) aludem que costumam decorrer situações de agressão física, verbal e sexual nos balneários. A orientação sexual e a aparência física são as principais causas de exclusão e assédio nas aulas de educação física, existindo um determinado sexismo e preconceito homofóbico no desporto em geral, mais especificamente na disciplina de educação física (Gill et al., 2010). 
Perante esta realidade consideramos interessante a elaboração do presente estudo que tem como objetivo, explorar e identificar a perceção de homofobia interna e de discriminação quotidiana da comunidade LGBTQIA+ no contexto da disciplina de educação física.

\section{Método}

Estudo quantitativo, qualitativo e exploratório numa amostra por conveniência, não probabilística.

\section{Participantes}

Participaram no presente estudo, 180 indivíduos da comunidade LGBTQIA+ com uma idade compreendida entre os 18 e 60 anos $(M=32,18$; $D P=11,56)$. De todos participantes, 63,3\% $(n=114)$ identificaram-se como sendo do sexo masculino, 34,4\% ( $n=62)$ sexo feminino e 2,2\% $(n=4)$ consideramse não binário. A orientação sexual dos participantes foi maioritariamente homossexual $(n=126)$, seguido de bissexual $(n=51)$, em que somente três participantes se consideraram assexuais. Relativamente às habilitações literárias dos participantes, $58,9 \%(n=128)$ referem ter concluído um curso superior, seguido do ensino secundário (26,7\%) enquanto 2,2\% tinha equivalência inferior ao ensino secundário e 12,2\% qualificação técnica superior profissional. Questionados sobre as memórias relacionadas com algum tipo de discriminação devido à orientação sexual no contexto da disciplina de educação física, $63,3 \%(n=114)$ referiram não ter sido alvo de discriminação, 34,4\% $(n=62)$ sentiram-se agredidos verbalmente e 2,2\% ( $n$ = 4) foram alvo de algum tipo de agressão física.

\section{Instrumentos}

\section{Questionário Sociodemográfico}

Foi utilizado um Questionário Sociodemográfico para obter um conjunto de informações sobre os participantes, tais como a idade, género com que se identificava, habilitações literárias, situação profissional e grau de concordância em relação às matérias do currículo nacional de educação física (de acordo com os Programas Nacionais de educação física disponíveis no Ministério da Educação ${ }^{1}$ ). No presente questionário foram realizadas também questões acerca do bullying, discriminação, exercício físico e as vivências em relação à disciplina de educação física, a saber: "No meio escolar, alguma vez foi vítima de discriminação devida à sua orientação sexual?"; "A discriminação veio por parte de quem?"; "Alguma vez foi alvo de discriminação devido à sua orientação sexual em contexto da disciplina de Educação Física?"; "Em relação à disciplina de Educação Física, alguma vez se sentiu retraído durante as aulas? (ex: não deu o seu melhor com receio de revelar a sua orientação sexual)"; "No que diz respeito à prática de exercício, ou prática de uma modalidade, alguma vez se sentiu menos capaz, devido à sua orientação

1 http://www.dge.mec.pt/educacao-fisica 
sexual?"; "Qual a modalidade que se sente menos capaz de praticar devido à sua orientação sexual?" e "Indique pelo menos três comentários que considera mais discriminatórios nas aulas de Educação Física".

\section{Questionário Conforto com a Identidade Sexual (Escala de Homofobia Internalizada)}

O Questionário Conforto com a Identidade Sexual [Internalized Homophobia Scale, versão original de Ross e Rosser (1996); tradução e validação para a população portuguesa por Pereira e Leal (2005)] permite a medição da homofobia internalizada. O questionário integra 26 itens tipo Likert com cinco opções de resposta ( 1 = discordo totalmente, 2 = discordo moderadamente, 3 = não discordo, nem concordo, 4 = concordo moderadamente, 5 = concordo totalmente) e agrupa duas dimensões: i) perceção interna do estigma associado à homossexualidade; ii) perceção externa do estigma associada à homossexualidade. $O$ coeficiente de fiabilidade do alfa de Cronbach da versão original portuguesa apresentou um índice de consistência interna apropriado ( $a=0,74$ ) (Marôco \& Garcia-Marques, 2006).

\section{Escala de Discriminação Quotidiana}

A Escala de Discriminação Quotidiana [Everyday Discrimination Scale, versão original de Williams et al. (1997), tradução e validação por Freitas et al. (2015)], avalia e compara a perceção e discriminação entre indivíduos quer na sua etnia quer orientação sexual. A escala apresenta oito itens numa escala de tipo Likert com seis opções de resposta [ 0 = nunca; 1 = raramente (menos de 1 vez/ano), 2 = por vezes (algumas vezes/ano), 3 = algumas vezes (algumas vezes/mês), 4 = muitas vezes (pelo menos uma vez/semana) e 5 = quase sempre (quase todos os dias)]. A escala apresenta dois fatores: Tratamento injusto $(\alpha=0,75)$ e Rejeição pessoal $(a=0,74)$, com índices de consistência interna também considerados aceitáveis.

\section{Procedimentos}

A presente amostra $(N=180)$ foi recolhida através de um questionário on-line estruturado no software de formulários da Google. Através da plataforma criou-se um link, que foi disponibilizado e divulgado em diversos grupos e páginas das redes sociais (e.g., Facebook e Instagram). Foi também solicitada e aceite a divulgação do presente estudo através do site oficial da associação ILGA Portugal - Intervenção Lésbica, Gay, Bissexual, Trans e Intersexo. De forma e melhor analisar a variável idade, criaram-se três classes de idades [adolescência (até 20 anos), adulto jovem (20-40 anos) e meia-idade (40-60 anos)] conforme o ciclo de desenvolvimento humano (Gonçalves, 2016).

A recolha da amostra decorreu entre o período de maio de 2021 e julho de 2021 . O estudo obedeceu a todos os procedimentos éticos, representados no consentimento de informação, participação livre e espontânea, tendo sido assegurada a utilização dos dados recolhidos, única e exclusivamente para efeitos do presente estudo. Foram garantidos a confidencialidade e anonimato dos participantes.

\section{Análise de Dados}

Optou-se pela técnica de análise de conteúdo para a representação da informação qualitativa (respostas abertas), tendo-se procedido à criação de categorias e subcategorias que foram analisadas com recurso ao software QDA Miner Lite. Foi utilizado um teste de fidelidade intraindividual (codificação utilizada por 
apenas um dos investigadores). Assim a partir da análise de conteúdo utilizada, ponderou-se a percentagem resultante do acordo entre duas codificações realizadas com um espaço temporal de uma semana. Os índices de fidelidade foram calculados com recurso à fórmula de Bellack (Índice de Fiabilidade

$=\frac{\text { Número de acordos }}{\text { Número de acordos+Número de desacordos }}$ X 100) (Bellack et al., 1966, como citado em Vieira, 2007, p. 174). A informação quantitativa foi analisada através do software IBM SPSS Statistics (versão 27), tendo-se analisado estatísticas descritivas, fiabilidade das escalas utilizadas, coeficientes de correlação e diferenças nas variáveis dependentes pelas independentes. Calculou-se o coeficiente de Spearman $\left(-1 \leq r_{S} \leq 1\right)$, para analisar correlações entre as variáveis. Calcularam-se os teste Wilcoxon-Mann-Whitney (U) e KruskalWallis $(H)$ para analisar as diferenças nas variáveis em estudo entre as categorias de variáveis independentes, por não se verificar o pressuposto da normalidade [Shapiro-Wilk $\left(S W_{(180)} H_{\text {Homofobia }}\right.$ internalizada $=0,85 ; p<0,001 ; S k=-1,72 ; K u=3,90) ;\left(S W_{(180)}\right.$ Discriminação Quotidiana $=0,84 ; p<0,001$; $S k=1,51 ; K u=2,35)]$.

\section{Resultados e Discussão}

Através do cálculo dos coeficientes alfa de Cronbach foi possível verificar que a fiabilidade das escalas foi aceitável para o questionário de Conforto com a Identidade Sexual (homofobia interna) (a de Cronbach = 0,76) e elevada fiabilidade para a Escala de Discriminação Quotidiana ( $a$ de Cronbach $=0,92$ ) e respetivas dimensões (Tratamento injusto, a de Cronbach =0,91; Rejeição Pessoal, $a=0,90$ ) (Marôco \& GarciaMarques, 2006). Os resultados de fiabilidade das escalas no presente estudo apresentaram valores de fiabilidade superiores quando comparados com as validações dos estudos originais (Freitas et al., 2015; Pereira \& Leal, 2005). O Índice de Fiabilidade para a análise qualitativa apresentou um valor de $98,4 \%$ (acordo intraobservador); foi ainda calculado o índice de fidelidade interobservador (97,4\%) resultante da codificação realizada por dois investigadores.

Verificadas a intensidade de associação entre a Homofobia Internalizada, a Discriminação Quotidiana e as variáveis relacionadas com a disciplina de Educação Física, a Tabela 1 apresenta correlações de intensidade fraca a intensidade moderada [fracas $<0,25$; moderadas $0,25 \leq\left|r_{\mathrm{s}}\right|<0,5$; fortes $0,5 \leq\left|r_{\mathrm{S}}\right|<0,75$; muito fortes $\left|r_{s}\right|>0,75$ (Marôco, 2018, p. 23)]. De realçar que na avaliação da homofobia internalizada, somente a Perceção de estigma externo apresentou correlações moderadas e estatisticamente significativas com as variáveis "Discriminação", "Vítima de discriminação no meio escolar", "Avaliação da disciplina de Educação Física segundo as vivências", "Vítima de discriminação na disciplina de educação física", "Retração na disciplina de educação física", "Prática atual das modalidades que gostava" e "Sentimentos de capacidade de realizar modalidade". Por outro lado, verificou-se uma correlação moderada e positiva entre a variável Discriminação Quotidiana e as variáveis "Vítima de discriminação no meio escolar", "Vitima de discriminação na disciplina de educação física" e "Retração na disciplina de Educação Física". 


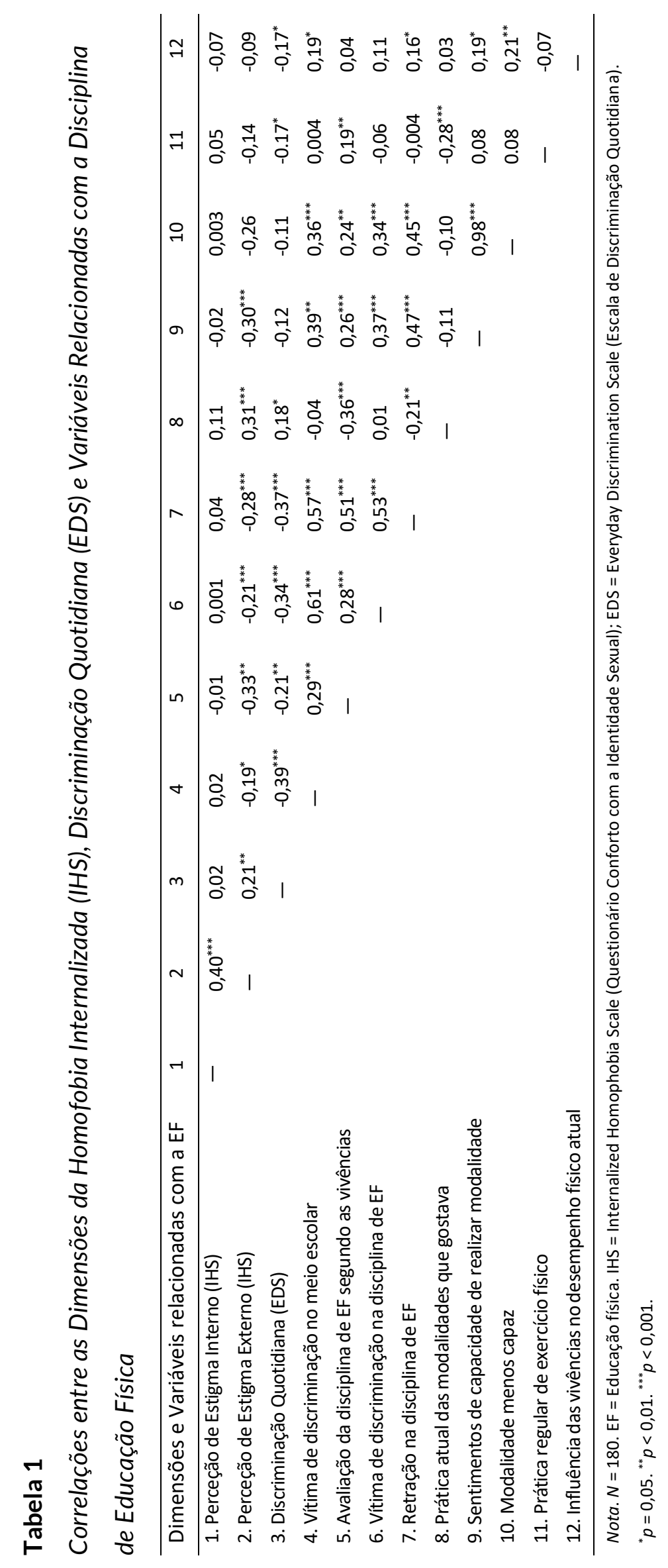


Tais correlações podem dever-se ao facto de os participantes em estudo terem memórias de carácter discriminatório aquando a prática da disciplina de educação física. Por exemplo, ao se analisar a questão aberta sobre os comentários considerados mais discriminatórios pelos participantes na aula de educação física, surgiram sentimentos que se relacionaram com a prática de bullying. Assim, na categoria bullying, a análise de conteúdo revelou subcategorias em que os participantes demonstraram unidades de análise consideradas como as mais discriminatórias (Figura 1), tais como: Humilhação ("Aprende a jogar, ó

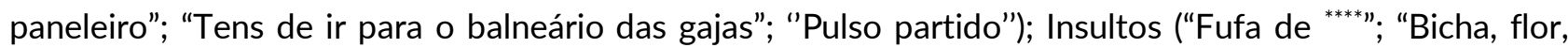
gay"; "Fufa, machona"); Body shaming ("Olha aquela gorda está aqui e não faz nada"; "Monte de banhas"; "Gordo/a") e Ameaças ("Vais apanhar no balneário").

\section{Figura 1}

Subcategorias de Discriminação Relacionadas ao Bullying

\section{Humillhação}

Ameaças Body shaming Insultos

Nota. $N=98$. Subcategorias de discriminação consideradas mais relevantes pelos participantes: Humilhação $(54,9 \%)$, Insultos (9,9\%), Bodyshaming (crítica sobre a aparência física) (5,6\%), Ameaças (0,6\%).

Uma análise cruzada de ambas as metodologias (quantitativa vs. qualitativa) permitiu verificar que os participantes LGBTQIA+ percecionam algum tipo de vitimização e discriminação em meio escolar e que esta discriminação também pode ser vivenciada nas aulas de educação física. À semelhança dos resultados apresentados no nosso estudo, Berg e Kokkonen (2021) revelam que grande parte dos estudantes já presenciou situações de discriminação em meio escolar que envolvem indivíduos da comunidade LGBTQIA+. De facto, vários estudos têm evidenciado situações em que a orientação sexual pode ser uma das principais causas de exclusão e assédio nas aulas de educação física (Gill et al., 2010), e que o preconceito homofóbico e sexismo estão evidentes na disciplina de educação física e no desporto (Landi et al., 2020; Rosa et al., 2020). A discriminação relacionada com a homossexualidade na disciplina de educação física, pode ser principalmente experienciada pelos alunos através da violência verbal (e.g., humilhação, insultos, ameaças) (Gill et al., 2010; Landi et al., 2020; Rosa et al., 2020).

Numa perspetiva similar e em conformidade com os resultados encontrados no presente estudo, em que se questionou sobre a recordação de os participantes terem vivenciado algum tipo de discriminação nas aulas de educação física, Prado (2017) revela que alunos LGBTQIA+ têm a tendência a ficar no silêncio ou a participarem menos nas aulas de forma a evitarem insultos relacionados com a sua orientação sexual. Assim, pode ser espectável que a não participação nas aulas de educação física possa ser comum, uma vez que se manifesta 
falta de empenho e uma diminuição no interesse dos alunos pela disciplina de Educação Física e na prática de atividade física (Rodrigues, 2018).

Se se tiver em consideração as correlações negativas entre a dimensão Perceção de estigma externo e as modalidades que os participantes gostavam de praticar [Futebol $\left(r_{S}=-0,22 ; p<0,01\right)$, Voleibol $\left(r_{S}=-0,18\right.$; $p<0,01)$, Basquetebol $\left(r_{s}=-0,18 ; p<0,01\right)$, Andebol $\left(r_{s}=-0,23 ; p<0,01\right)$, Ginástica $\left(r_{s}=-0,30 ; p<0,001\right)$, Atletismo ( $\left.r_{s}=-0,27 ; p<0,001\right)$, Combate $\left(r_{S}=-0,33 ; p<0,001\right)$, Atividades Rítmicas Expressivas ( $r_{s}=-$ 0,30; $p<0,001)$, Jogos Tradicionais $\left(r_{s}=-0,22 ; p<0,01\right)$ e Exploração da Natureza $\left(r_{s}=-0,25 ; p<0,01\right)$, é possível verificar que a perceção de estigma externo pode ter uma determinada influência sobre a modalidade que os participantes gostavam de praticar ou praticam. De facto, Greenspan et al. (2019) revelam a existência de uma discrepância entre a prática de determinadas modalidades existentes na disciplina de educação física, em que a comunidade LGBTQIA+ evita frequentemente as aulas de educação física e balneários devido à sensação de desconforto.

Através de estatística inferencial também foi possível verificar diferenças estatisticamente significativas na modalidade que gostava de praticar durante as aulas de educação física entre os sexos (masculino, feminino e não binário). Estas diferenças verificaram-se para a modalidade de Futebol $\left(\chi_{(10)}^{2}=35,53 ; p<0,001\right)$, Voleibol $\left(\chi_{(10)}^{2}=27,84 ; p=0,002\right)$, Basquetebol $\left(\chi_{(10)}^{2}=16,08 ; p=0,041\right)$, Corfebol $\left(\chi_{(10)}^{2}=20,49 ; p=0,025\right)$, Raquetes $\left(\chi_{(10)}^{2}=31,63 ; p<0,001\right)$, e Combate $\left(\chi_{(10)}^{2}=25,26 ; p=0,005\right)$, em que o sexo feminino referiu gostar mais das modalidades referidas quando comparado com o sexo masculino. Acentuando-se que os participantes do sexo feminino estão em menor número. Não se verificaram diferenças estatísticas para as modalidades Andebol $\left(\chi_{(10)}^{2}=15,61 ; p=0,111\right)$, Ginástica $\left(\chi_{(10)}^{2}=5,84 ; p=0,829\right)$ e Atletismo $\left(\chi_{(10)}^{2}=18,22\right.$; $p=0,051)$.

Uma vez mais, através da metodologia qualitativa (Figura 2), foi possível analisar que o género foi a unidade de análise alvo de maior comentário discriminatório (e.g., "És rapariga não podes jogar", "As raparigas não sabem jogar", "Os rapazes preferem futebol", "Algum rapaz pode me ajudar a levar estas coisas pesadas?"), seguido da Modalidade (e.g., "Futebol é para rapazes", "Ginástica acrobática é para as meninas", "Vólei não é para rapazes") e Orientação Sexual (e.g., "Assumirem que a minha falta de capacidade desportiva é ligada à orientação sexual", "És gay por isso não jogas futebol", "Faz-te homem", "Joga futebol, é lésbica").

Os resultados revelaram que os participantes recordaram algum tipo de discriminação, corroborando com o estudo de Castro e Siqueira (2021) que revelaram existir um clima hostil no que diz respeito à orientação sexual no mundo do desporto. Segundo estes autores, este ambiente hostil pode estar associado a estereótipos relacionados com a dicotomia género-modalidade.

A associação de determinadas modalidades aos sexos, tais como o futebol estar associado aos rapazes e às raparigas se associarem modalidades sem contacto, tais como a ginástica e a dança (Chalabaev et al., 2013), também podem influenciar o ambiente vivenciado pelos indivíduos nas aulas de educação física. Por outro lado, a adequação e gosto pelas diferentes modalidades desportivas relacionadas aos sexos, podem estar associadas à visão de fragilidade quando se referem ao sexo feminino e a virilidade quando se referem ao sexo masculino (Mariano \& Altmann, 2016). Um estudo de Peçanha e Devide (2010) revela a possibilidade de existir um determinado preconceito associado ao género e tipo de atividade física (consequentemente às habilidades motoras de base), independentemente da orientação sexual. Tal 
preconceito, pode dever-se também ao facto de o significado de género sofrer algumas alterações com o evoluir da sociedade (Gonçalves \& Gonçalves, 2021).

\section{Figura 2}

Subcategorias de Discriminação na Educação Consideradas Não-Bullying

\section{Modalidade Orientação Sexual}

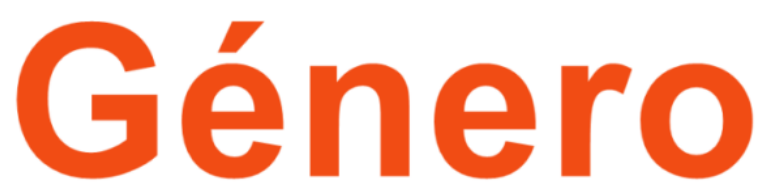

Nota. $N=98$. Subcategorias de discriminação consideradas mais relevantes pelos participantes como não-bullying: Género (11,1\%), Modalidade (9,9\%), Orientação Sexual (8,0\%).

Um estudo de Rodrigues (2018) revela que a modalidade de futebol surge como "grande vilão" dos alunos homossexuais, tal como o andebol, corroborando com os resultados apresentados neste estudo, em que também se evidenciou que os participantes do sexo feminino referem a modalidade de futebol como a que mais gostavam de praticar. Contrariamente, Flintoff e Scraton (2006) mencionam que rapazes e raparigas apresentam preferências diferentes nas matérias do currículo de educação física, isto é, os rapazes têm um comportamento e uma atitude mais positiva em relação a jogos desportivos e enquanto que as raparigas apresentam maior preferência pela dança.

Analisada a variável da Classe de idades através da realização do teste Kruskal-Wallis, foi possível verificar diferenças estatisticamente significativas na "Perceção de Estigma Externo" $\left(H_{(2)}=7,62 ; p=0,02 ; N=180\right)$. De acordo com a comparação múltipla de médias das ordens, a classe de idades dos Adolescentes (Posto Médio $=112,12$ ) apresentou uma média superior na variável da Perceção de estigma externo significativamente diferente da classe de idades Meia-idade (Posto Médio = 78,66) e Adulto jovem (Posto Médio $=90,15)$. Estudos revelam existir diferenças de discriminação nas várias fases do desenvolvimento humano (Anhalt et al., 2020; Badgett et al., 2019; Parmenter et al., 2021). No entanto, a investigação nesta área não parece ser consensual. Um estudo de Van Beusekom et al. (2018) não revelou diferenças na homofobia internalizada segundo a idade e, no entanto, Lee et al. (2019) afirmam que os jovens apresentam, um maior sentimento de homofobia internalizada. Perante estas divergências são recomendados estudos futuros.

Analisadas as dimensões de homofobia internalizada (Perceção do estigma interna e Perceção do estigma externo) e a avaliação da disciplina de educação física conforme as vivências escolares dos participantes, verificaram-se diferenças estatisticamente significativas para a dimensão Perceção de estigma externo $\left(H_{(2)}\right.$ = 20,17; $p<0,001 ; N=180$ ). Os participantes que Detestavam (Posto Médio = 111,82) e Não Gostavam (Posto Médio $=108,13$ ) da disciplina de educação física, apresentaram uma distribuição da Perceção de estigma significativamente diferente dos participantes que referiram Indiferença (Posto Médio $=85,29$ ), 
Gostar (Posto Médio = 85,26) e Adorar (Posto Médio = 64,98) da disciplina de Educação Física. Através dos resultados no presente estudo verificaram-se diferenças estatisticamente significativas que revelam descontentamento face à disciplina de educação física quando comparada com a variável Perceção do estigma externo. De facto, Rodrigues (2018) e Symons et al. (2010) salientam a vivência de situações traumáticas (e.g., humilhação, insultos, ameaças) que podem levar ao desinteresse ou abandono da prática de atividade física. Segundo estes autores, a perceção das habilidades motoras e desportivas são apontadas como um indicador de os indivíduos que apresentam estas características (habilidades motoras) terem preferência pela disciplina de educação física. O estudo de Pereira e Leal (2005) menciona a presença de homofobia na própria comunidade LGBTQIA+, podendo esta manifestar-se através de homofobia internalizada, subdividida por perceção de estigma externo ou interno.

As variáveis Homofobia internalizada (dimensão Perceção de Estigma Externo) e Discriminação Quotidiana foram comparadas entre os níveis de retração dos participantes nas aulas de educação física, tendo sido reveladas diferenças estatisticamente significativas.

\section{Tabela 2}

Diferenças na Perceção de Estigma Externo, Discriminação Quotidiana quanto à Retração na Disciplina de Educação Física

\begin{tabular}{|c|c|c|c|c|}
\hline Dimensão e Variável & Retração na disciplina de Educação Física & Posto médio & $H(2)$ & $p$ \\
\hline \multirow{5}{*}{ Perceção de Estigma Externo (IHS) } & Sim, com muita frequência & 128,22 & \multirow{5}{*}{16,19} & \multirow{5}{*}{0,003} \\
\hline & Sim, com alguma frequência & 101,35 & & \\
\hline & Sim, ocasionalmente & 93,20 & & \\
\hline & Sim, raramente & 97,03 & & \\
\hline & Nunca & 77,98 & & \\
\hline \multirow{5}{*}{ Discriminação Quotidiana (EDS) } & Sim, com muita frequência & 124,11 & \multirow{5}{*}{27,93} & \multirow{5}{*}{$<0,001$} \\
\hline & Sim, com alguma frequência & 114,13 & & \\
\hline & Sim, ocasionalmente & 94,70 & & \\
\hline & Sim, raramente & 112,08 & & \\
\hline & Nunca & 71,82 & & \\
\hline
\end{tabular}

Nota. $N=180$. IHS = Dimensão da Internalized Homophobia Scale (Questionário Conforto com a Identidade Sexual); EDS = Everyday Discrimination Scale (Escala de Discriminação Quotidiana).

${ }^{* * *} p<0,001$.

Através do teste Wilcoxon-Mann-Whitney, analisaram-se as diferenças estatísticas na Homofobia internalizada e Discriminação quotidiana segundo as modalidades que os participantes mais praticavam nas aulas de educação física. Verificaram-se diferenças estatisticamente significativas na dimensão Perceção de estigma externo $\left(U=5429,5 ; p_{u}<0,001, N=180\right)$ e na variável Discriminação quotidiana $(U=$ 4839,5; $p_{u}=0,014, N=180$ ) entre os participantes que praticavam e não praticavam atualmente as modalidades. Isto é, os participantes que percecionaram estigma externo e discriminação foram os 
participantes que revelaram não praticar qualquer modalidade na atualidade. Estes resultados são corroborados pelo estudo de Symons et al. (2010) que mencionam a prática das modalidades na disciplina de educação física e desporto estão relacionadas com a predisposição para a prática de exercício físico na idade adulta. Tendo esta premissa em consideração e à semelhança dos resultados obtidos no presente estudo, Denison e Kitchen (2015) relatam que a comunidade LGBTQIA+ tem tendência a não participar em clubes ou equipas desportivas, devido às vivências anteriores nas aulas de educação física. Estes autores salientam ainda que as mulheres lésbicas temem ser discriminadas devido às suas competências e habilidades motoras, enquanto que os homens gays sentem maior receio de ser discriminados devido à sua sexualidade. De acordo com Baiocco et al. (2018), os homens homossexuais desistem com mais frequência da prática desportiva devido a algum tipo de insulto, receio de se sentirem discriminados e a sentirem-se pressionados socialmente a frequentarem e praticarem modalidades consideradas masculinas.

\section{Conclusões}

Uma escola perfeita seria um local onde reina a partilha de conhecimentos, experiências e valores, onde o sentido crítico e o debate fossem desenvolvidos e a amizade e empatia predominasse. No entanto, esta é uma realidade que ainda não pode ser reivindicada. Em ambiente escolar são cada vez mais recorrentes situações de bullying entre alunos. Estas ocorrências são frequentes principalmente contra minorias (etnia, religião, orientação sexual, estatuto socioeconómico). No que concerne à orientação sexual, o presente estudo permitiu verificar que os participantes em estudo, têm memórias em que ocorreram situações de discriminação no contexto escolar e no decorrer das aulas da disciplina de educação física.

Neste contexto são vivenciados momentos de violência verbal causados devido a algum tipo de estereotipização de determinados géneros a determinadas modalidades desportivas, que quando fogem da norma estabelecida pela sociedade em que os participantes se inserem, são alvo de marginalização. Neste sentido os participantes LGBTQIA+ tendem a não se identificar com a disciplina de educação física e consequentemente podem perder o interesse pela prática de atividade física na fase adulta, tal como os resultados nos revelam.

De realçar que foi possível verificar uma diferenciação da prática das modalidades na disciplina de educação física entre o sexo. As participantes do sexo feminino têm preferência pelas modalidades coletivas de contacto, enquanto os participantes do sexo masculino revelam maior preferência por modalidades individuais ou coletivas sem contacto.

As limitações deste estudo prendem-se pelo facto de se analisarem as vivências de antigos alunos na disciplina de educação física, existindo um hiato muito grande entre o momento desta vivência e a atualidade. Acresce ainda a discrepância entre a idade mínima (18 anos) e idade máxima (60 anos), que resultam de vivências geracionais muito diversificadas em relação às reformas do sistema educativo, não sendo possível analisar esta variável no estudo.

Através deste estudo e respetiva revisão de literatura é possível verificar a perceção de homofobia por partes dos praticantes da disciplina de educação física. Numa outra perspetiva, estudos futuros devem explorar qual a perceção de homofobia externa por partes de todos os intervenientes na elaboração do 
currículo nacional de educação física, tendo em consideração a evolução do conceito LGBTQIA+ na sociedade atual.

Agradecimentos | Acknowledgements: Nenhum | None.

Conflito de interesses | Conflict of interest: Nenhum | None.

Fontes de financiamento | Funding sources: Nenhuma | None.

Contributos: ACC: Redação do manuscrito; Recolha e tratamento dos dados; Análise e discussão dos dados; Revisão do manuscrito; JM: Recolha e tratamento dos dados; Redação e revisão do manuscrito; FV: Tratamento dos dados; redação e revisão do manuscrito.

\section{Referências}

American Psychological Association. (2012). Guidelines for psychological practice with lesbian, gay, and bisexual clients. American Psychologist, 67(1), 10-42. https://doi.org/b32c63

Anhalt, K., Toomey, R. B., \& Shramko, M. (2020). Latinx sexual minority youth adjustment in the context of discrimination and internalized homonegativity: The moderating role of cultural orientation processes. Journal of Latinx Psychology, 8(1), 41-57. https://doi.org/gk4gqn

Badgett, M. V. L., Waaldijk, K., \& Rodgers, Y. van der M. (2019). The relationship between LGBT inclusion and economic development: Macro-level evidence. World Development, 120, 1-14. https://doi.org/gg3gm4

Baiocco, R., Pistella, J., Salvati, M., loverno, S., \& Lucidi, F. (2018). Sports as a risk environment: Homophobia and bullying in a sample of gay and heterosexual men. Journal of Gay \& Lesbian Mental Health, 22(4), 385411. https://doi.org/d5fz

Barounis, C. (2017). "Not the Usual Pattern": James Baldwin, homosexuality, and the DSM. Criticism, 59(3), 395415. https://doi.org/g5f9

Berg, P., \& Kokkonen, M. (2021). Heteronormativity meets queering in physical education: The views of PE teachers and LGBTIQ+ students. Physical Education and Sport Pedagogy, 1-14. https://doi.org/g5gb

Beserra, J. T. S., Brito, A. K. A., \& Ribeiro, S. L. G. (2019). Homofobia nas aulas de educação física: um desafio para os professores de educação física do município de buriti dos montes - Piauí. Revista Form@re, 7(2), 9190. https://bit.ly/3FOMsfC

Borrillo, D. (2009). A homofobia. Em T. Lionço \& D. Diniz (Eds.), Homofobia e educação. Letras Livres. https://bit.ly/3D3OB9N

Castro, G. H. C. D., \& Siqueira, M. V. S. (2021). "Vão achar que é uma piada, mas, para nós, não!": Discursos de resistência em clubes brasileiros de futebol gay. Cadernos EBAPE.BR, 18, 1058-1070. https://doi.org/g5gc

Chalabaev, A., Sarrazin, P., Fontayne, P., Boiché, J., \& Clément-Guillotin, C. (2013). The influence of sex stereotypes and gender roles on participation and performance in sport and exercise: Review and future directions. Psychology of Sport and Exercise, 14(2), 136-144. https://doi.org/f4pd9n

Denison, E., \& Kitchen, A. (2015). Out on the fields: The first international study on homophobia in sport. Nielsen Sport, Bingham Cup 2014, Sydney Convicts, Sport Australia. https://bit.ly/3HvvGXZ

Devís-Devís, J., Pereira-García, S., López-Cañada, E., Pérez-Samaniego, V., \& Fuentes-Miguel, J. (2018). Looking back into trans persons' experiences in heteronormative secondary physical education contexts. Physical Education and Sport Pedagogy, 23(1), 103-116. https://doi.org/gm5tkr

Drescher, J. (2015). Out of DSM: Depathologizing homosexuality. Behavioral Sciences, 5(4), 565-575. https://doi.org/10.3390/bs5040565

Flintoff, A., \& Scraton, S. (2006). Girls and physical education. Em D. Kirk, D. MacDonald, \& M. O'Sullivan (Eds.), Handbook of physical education (pp. 767-783). SAGE Publications Ltd. https://doi.org/g5gd 
Freitas, D. F., Coimbra, S., Marturano, E. M., \& Fontaine, A. M. (2015). Adaptação da escala de discriminação quotidiana para jovens portugueses. Psicologia: Reflexão e Crítica, 28(4), 708-717. https://doi.org/gf646m

Gill, D. L., Morrow, R. G., Collins, K. E., Lucey, A. B., \& Schultz, A. M. (2010). Perceived climate in physical activity settings. Journal of Homosexuality, 57(7), 895-913. https://doi.org/fc954s

Gonçalves, J. P. (2016). Ciclo vital: Início, desenvolvimento e fim da vida humana. Possíveis - Contribuições para educadores. Contexto \& Educação, 31(98), 79-110. https://bit.ly/31QOjp5

Gonçalves, M. C., \& Gonçalves, J. P. (2021). Gênero, identidade de gênero e orientação sexual: Conceitos e determinações de um contexto social. Revista Ciências Humanas, 14(1), 1-6. https://doi.org/g5gf

Greenspan, S. B., Griffith, C., Hayes, C. R., \& Murtagh, E. F. (2019). LGBTQ + and ally youths' school athletics perspectives: A mixed-method analysis. Journal of LGBT Youth, 16(4), 403-434. https://doi.org/gg9g3b

Herrick, S. S. C., \& Duncan, L. R. (2020). Locker-room experiences among LGBTQ+ adults. Journal of Sport and Exercise Psychology, 42(3), 227-239. https://doi.org/gm5tkn

Hossain, F., \& Ferreira, N. (2019). Impact of social context on the self-concept of gay and lesbian youth: A systematic review. Global Psychiatry, 2(1), 51-78. https://doi.org/ghzqc2

Landi, D., Flory, S. B., Safron, C., \& Marttinen, R. (2020). LGBTQ Research in physical education: A rising tide? Physical Education and Sport Pedagogy, 25(3), 259-273. https://doi.org/g5gg

Lee, H., Operario, D., Yi, H., Choo, S., \& Kim, S.-S. (2019). Internalized Homophobia, depressive symptoms, and suicidal ideation among lesbian, gay, and bisexual adults in South Korea: An age-stratified analysis. LGBT Health, 6(8), 393-399. https://doi.org/g5gh

Macedo, A. (2018). Identidade de género e orientação sexual na prática clínica. Edições Sílabo.

Mariano, M., \& Altmann, H. (2016). Educação física na educação infantil: Educando crianças ou meninos e meninas? Cadernos Pagu, 46, 411-438. https://doi.org/g5gj

Marôco, J. (2018). Análise estatística com o SPSS Statistics ( $7^{\mathrm{a}}$ ed.). Report Number.

Marôco, J., \& Garcia-Marques, T. (2006). Qual a fiabilidade do alfa de Cronbach? Questões antigas e soluções modernas? Laboratório de Psicologia, 4(1), 65-90. https://doi.org/10.14417//p.763

Nadal, K. L., Wong, Y., Issa, M.-A., Meterko, V., Leon, J., \& Wideman, M. (2011). Sexual orientation microaggressions: Processes and coping mechanisms for lesbian, gay, and bisexual individuals. Journal of LGBT Issues in Counseling, 5(1), 21-46. https://doi.org/btnf6m

Parmenter, J. G., Galliher, R. V., \& Maughan, A. D. A. (2021). LGBTQ+ emerging adults perceptions of discrimination and exclusion within the LGBTQ+ community. Psychology \& Sexuality, 12(4), 289-304. https://doi.org/ggj9tw

Peçanha, M. B., \& Devide, F. P. (2010). A prática pedagógica em relação ao bullying homofóbico nas aulas de educação física escolar: $O$ discurso dos docentes do primeiro segmento do ensino fundamental [Apresentação de artigo]. Fazendo Gênero 9. Diásporas, Diversidades, Deslocamentos, Universidade Federal de Sta. Catarina, Brasil. Anais Electrónicos, 1-12. https://bit.ly/3BXLDSQ

Pereira, H., \& Leal, I. P. (2005). A identidade (homo)sexual e os seus determinantes: Implicações para a saúde. Análise Psicológica, 23(3), 315-322. https://doi.org/10.14417/ap.95

Prado, V. M. (2017). Entre queerpos e discursos: Normalização de condutas, homossexualidades e homofobia nas práticas escolares da Educação Física. Praxis Educativa, 12(2), 501-519. https://doi.org/g5gk

Rocha, A., Rodrigues, I., Matos, J., \& Carmona, R. (2019). Relatório do Projeto Educação LGBTI 2019. Novembro 2020, 60. https://bit.ly/2YxMcol

Rodrigues, A. P. (2018). Que time é teu?": Um debate sobre homofobia nas aulas de Educação Física. Educação Física na Escola Básica: Debates contemporâneos, 113-136. https://bit.ly/3F5Ls9U

Rosa, M. V. da, Souza, M. D. O., \& Borges, A. M. (2020). Preconceito contra a mulher na educação física escolar no nono ano. Revista Prâksis, 1, 102. https://doi.org/g5gm

Ross, M. W., \& Rosser, B. R. S. (1996). Measurement and correlates of internalized homophobia: A factor analytic study. Journal of Clinical Psychology, 52(1), 15-21. https://doi.org/bhpq3p

Santos, J. J., \& Cerqueira-Santos, E. (2020). Homofobia e escola: Uma revisão sistematizada da literatura. Revista Subjetividades, 20(1), 1-14. https://doi.org/g5gn 
Santos, L. P. dos. (2017). Diversidade sexual e homofobia na escola: Posicionamentos de professoras e professores do ensino básico. Itinerarius Reflectionis, 13(2), 01-22. https://doi.org/g5gp

Symons, C., Sbaraglia, M., Hillier, L., \& Mitchell, A. (2010). Come out to play: The sports experiences of lesbian, gay, bisexual and transgender LGBT) people in Victoria. Institute of Sport, Exercise and Active Living: School of Sport and Exercise Science, Victoria University.

UNICEF. (2017). Gender equality. UNICEF Regional Office for South Asia; https://uni.cf/3HcRUhe

Van Beusekom, G., Bos, H. M., Kuyper, L., Overbeek, G., \& Sandfort, T. G. (2018). Gender nonconformity and mental health among lesbian, gay, and bisexual adults: Homophobic stigmatization and internalized homophobia as mediators. Journal of Health Psychology, 23(9), 1211-1222. https://doi.org/g5gq

Vieira, F (2007). As orientações educacionais dos professores de educação física e o currículo institucional. Instituto Piaget.

Williams, D. R., Yan Yu, Jackson, J. S., \& Anderson, N. B. (1997). Racial differences in physical and mental health: Socio-economic status, stress and discrimination. Journal of Health Psychology, 2(3), 335-351. https://doi.org/bzrgfb

Woodford, M. R., Howell, M. L., Kulick, A., \& Silverschanz, P. (2013). "That's so gay": Heterosexual male undergraduates and the perpetuation of sexual orientation microagressions on campus. Journal of Interpersonal Violence, 28(2), 416-435. https://doi.org/gg28nc 